\title{
SYNTHESIS OF STRUCTURALLY NOVEL CARBON MICRO/ NANOSPHERES BY LOW TEMPERATURE-HYDROTHERMAL PROCESS
}

\author{
KRISHNAMURTHY G ${ }^{* a}$ AND NAMITHA R $R^{b}$ \\ ${ }^{a, b}$ Department of Studies in Chemistry, Bangalore University, Bangalore-560 001, India
}

(Received: December 11, 2012 - Accepted: June 9, 2013)

\begin{abstract}
Carbon spheres with two to four attached lobes having axial, trigonal and tetrahedral morphology were synthesized at considerably low temperature of 200 ${ }^{\circ} \mathrm{C}$ by a hydrothermal reaction. The source of carbon used was sucrose in sulfuric acid medium. The product obtained was characterized by X-ray diffraction, Fourier transforms infrared and Raman spectroscopy, which have inferred us the graphitic nature of the product. Further Scanning and Transmission electron microscopic studies have revealed the spheres having attractive morphology with attached lobes and nodes like structures. The carbon spheres are having the size ranging from $200 \mathrm{~nm}$ to $4 \mu \mathrm{m}$. The spheres are solid balls exhibiting metallic luster. They possess surface area and pore volume of $2.956 \mathrm{~m}^{2} \mathrm{~g}^{-1}$ and $0.616 \mathrm{x} 10^{-2}$ $\mathrm{cm}^{3} \mathrm{~g}^{-1}$ respectively as obtained from Brunauer-Emmett-Teller method. The yield product obtained was about $70-80 \%$.
\end{abstract}

Keywords: Hydrothermal Process, Carbon Spheres, Spheres with lobes, Low Temperature Synthesis, Microscopic Techniques.

\section{INTRODUCTION}

The syntheses of various carbon nanostructures have inspired interest in carbon studies especially with regard to the synthesis of graphite and diamond. In nature they exists under highly contrasting conditions and attracted considerable attraction. As this novel form of carbon materials have attracted a great interest owing to unique properties, ${ }^{1-4}$ which include high specific surface area, good mechanical stability, chemical inertness, and porous nature with large pore volume. ${ }^{5}$ An important insight gained by the discovery of carbon nanostructures is that the carbon atoms can form pentagonal and heptagonal carbon rings, and the combination of these two basic structural units with the hexagonal carbon rings can form a variety of morphologies.

In recent years, carbon materials have attracted considerable attention because of their novel morphologies and specially physical and chemical properties, such as fullerenes, ${ }^{6}$ hollow and solid spheres, ${ }^{7-13}$ nanotubes,${ }^{14-18}$ onions, ${ }^{19-21}$ nanorods and nanocoils. ${ }^{22}$ Among these, carbon spheres are becoming increasingly important due to their promising applications in the fields of medicine, pharmaceutics, material science and paint industry. ${ }^{23}$ They can be used as photonic crystals, support for catalysts, ${ }^{24}$ anodes in secondary lithium ion batteries, lubricants, hydrogen storage media, ${ }^{25}$ and so on.

There are several methods which can be used to synthesize carbon spheres, such as chemical vapor deposition, arc plasma technique, pyrolysis of carbon sources, self-assembly template approach, reduction of supercritical carbon dioxide with bimetallic lithium and potassium, hydrothermal reaction, etc. However, these synthetic methods are complicated and time-consuming. Some of the methods require complicated equipments and rigorous conditions. ${ }^{26}$ In addition, the high cost of production limits their industrial fabrication in large quantities. These demerits have lead to discovery of new methods which presumably allow synthesizing highly pure carbon spheres in large scale, at lower cost and at relatively lower temperature. Therefore, the authors have explored a simple, moderately low temperature and pressure based hydrothermal technique to synthesize the elemental solid carbon.

The hydrothermal processes have great potential for the preparation of carbon particles with varying morphologies. The closed system, characterized by low temperature growth and a suitably tailored atmosphere to produce oxidizing or reducing conditions is an excellent way to produce carbon polymorphs. Thus, hydrothermal technique allows the synthesis of phases, which cannot be stabilized by any other method at such low pressure temperatures. This general approach provides an efficient, scalable route to synthesize spherical, porous carbon particles with porous structure and is suitable for large scale manufacturing. One frequently used technique to synthesize porous carbon materials involves a two-step templating approach, which requires infiltration of carbon precursor molecules into the pores of a preformed inorganic framework (e.g., mesoporous silica), carbonization of the precursor and removal of the inorganic template..$^{27,28}$

To date, mesoporous carbon particles with rod, fiber, plate, and donut-like morphologies were prepared by using mesoporous silica templates. Although this two-step templating technique allows for precise control of pore sizes and pore structures, it has several limitations such as incomplete filtration of the carbon precursor and formation of nanoporous carbon on the exterior of the template. In addition, it requires many tedious and time consuming infiltration steps. As an alternative approach, many researchers have focused on direct one-step methods. These methods generally lead to the formation of porous carbon in the form of monoliths or powders. Direct synthesis of porous carbon particles still remains challenging.

The present work aims to synthesize carbon spheres with attached lobes. Here, we report, an easy approach towards the synthesis of porous carbon spheres from sucrose using a direct one-step hydrothermal process. Interestingly, these carbon spheres are having two to four attached lobes having axial, trigonal and tetrahedral morphology. Probably there are no or a few reports on such carbon spheres with attached lobes and nodes, which were synthesized at $200^{\circ} \mathrm{C}$ by a hydrothermal procedure.

\section{EXPERIMENTAL}

\section{Characterization Techniques}

The as prepared products were characterized by X-ray powder diffraction (XRD) performed on a Bruker D8 Advance powder X-ray diffractometer to check the purity and phase structure of products, Fourier Transform Infrared (FTIR) using FTIR 8400S Shimadzu spectrophotometer and Raman spectral studies on FT-Raman (Perkin Elmer) instrument were made to understand the graphitic nature of the product. The surface morphology of the samples was examined with scanning electron microscope (SEM) using JEOL model JSM $6490 \mathrm{LV}$ and Transmission electron microscope (TEM), Phillips CM 12. Micomeritics BET Surface area analyzer model ASAP 2020 was used to determine the surface area and pore volume of the spheres.

\section{Synthesis of carbon spheres}

In a typical procedure, about $1 \mathrm{~g}$ of sucrose dissolved in $30 \mathrm{ml}$ of distilled water was acidified with sulfuric acid $(4.5 \mathrm{M})$. The solution of total $40 \mathrm{ml}$ was magnetically stirred for $30 \mathrm{~min}$ and then taken into a stainless steel autoclave with Teflon inner lining of $80 \mathrm{ml}$ capacity. The above autoclave, containing the raw materials was sealed tightly and kept into a digital electronic muffle furnace. Then it was heated to $200{ }^{\circ} \mathrm{C}$ for about $10 \mathrm{~h}$ and allowed to cool to room temperature. The black product obtained was collected by filtration and washed sequentially with anhydrous ethanol, hydrochloric acid, and deionized water several times to remove the residual impurity and other soluble matters. It was then oven dried at $100^{\circ} \mathrm{C}$ for $10 \mathrm{~h}$. The ultimate black product obtained was examined by various analytical techniques like XRD, FTIR, Raman spectroscopy, SEM, TEM, BET method.

\section{RESULTS AND DISCUSSION}

The phase and nature (crystalline or amorphous structure) of the obtained product was studied by XRD analysis, which was performed on Bruker D8 Advance powder X-ray diffractometer using the monochromatic highintensity $\mathrm{CuK} \alpha$ radiation $(\lambda=0.1541874 \mathrm{~nm})$. A typical XRD pattern of the as synthesized products is shown in Figure 1. There is a sharp peak at $2 \theta=20^{\circ}$ that 
corresponds to the (002) plane of graphite. In addition, a small shoulder peak at around $45^{\circ}$, which corresponds to the (001) plane of graphite. The sharpness of the peaks suggests the presence of crystalline carbon phase within the carbon spheres.

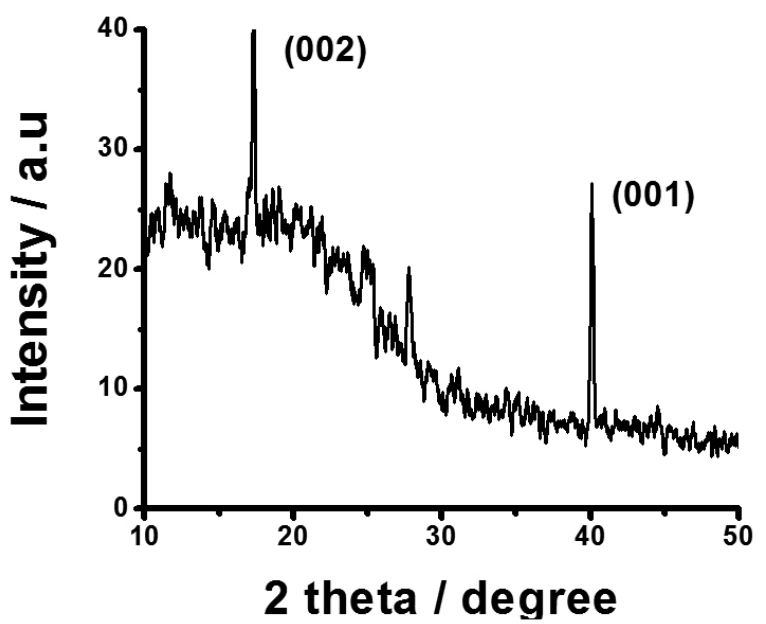

Figure. 1 is the XRD pattern of the as- synthesized carbon spheres in which the peak around $20^{\circ}$ indicating the (002) plane of the grapheme structures.

The FTIR and Raman spectroscopy were used to characterize these carbon structures using their vibrational frequencies. The FTIR spectrum in Figure 2 showing the characteristic peaks of $\mathrm{C}=\mathrm{C}$ of graphene materials in the range $1340-1700 \mathrm{~cm}^{-1}$. The absorption peak at $1118 \mathrm{~cm}^{-1}$ corresponds to the $\mathrm{C}-\mathrm{OH}$ stretching and $\mathrm{OH}$ bending vibrations, and the peaks at 2921 and $2852 \mathrm{~cm}^{-1}$ originate from the $\mathrm{C}-\mathrm{H}$ stretching vibrations. The peaks at 1580 and $1636 \mathrm{~cm}^{-1}$ are due to the stretching vibration of carboxylic group. These results indicate that there are a large number of residues including hydroxyl and carboxyl groups. They play important roles in the formation process of spherical structures. Furthermore they provide a potential avenue to load other molecules, ions, functional groups.

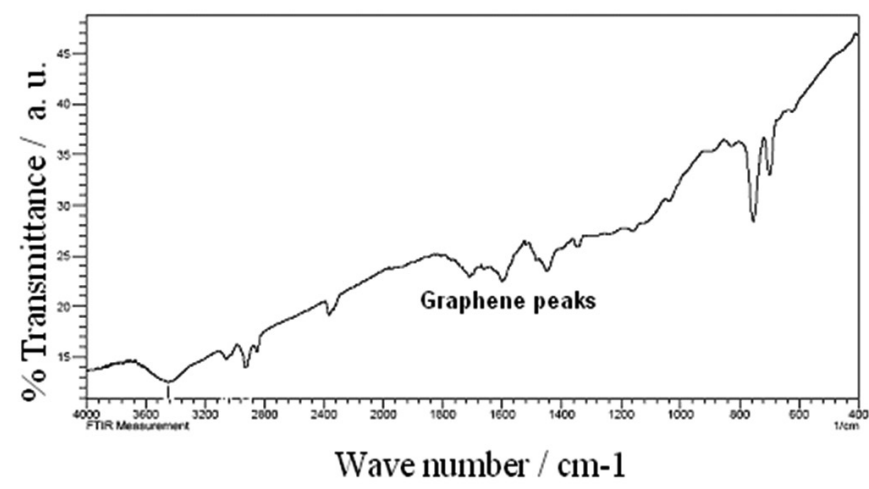

Figure. 2 FTIR spectrum of the as- synthesized carbon spheres showing the peaks in the range $1340-1700 \mathrm{~cm}^{-1}$ for graphene materials.

The Raman spectral run of the sample has also given the characteristic peaks corresponding to $\mathrm{C}=\mathrm{C}$ units in the product at 1360 and $1580 \mathrm{~cm}^{-1}$ as shown in Figure 3. The spectrum has the peak at $1380 \mathrm{~cm}^{-1}$ (D-Band), which corresponds to the vibrations of carbon atoms with dangling bonds in plane terminations of disordered graphite and the peak at $1580 \mathrm{~cm}^{-1}$ (G-Band) which can be attributed to the $\mathrm{E}_{2}$ mode of graphite. The other peaks correspond to amorphous carbon structure, with a high content of lattice edges or plane defects within the analyzed carbon spheres. The intensities of the peaks reveal the fairly good graphitization.

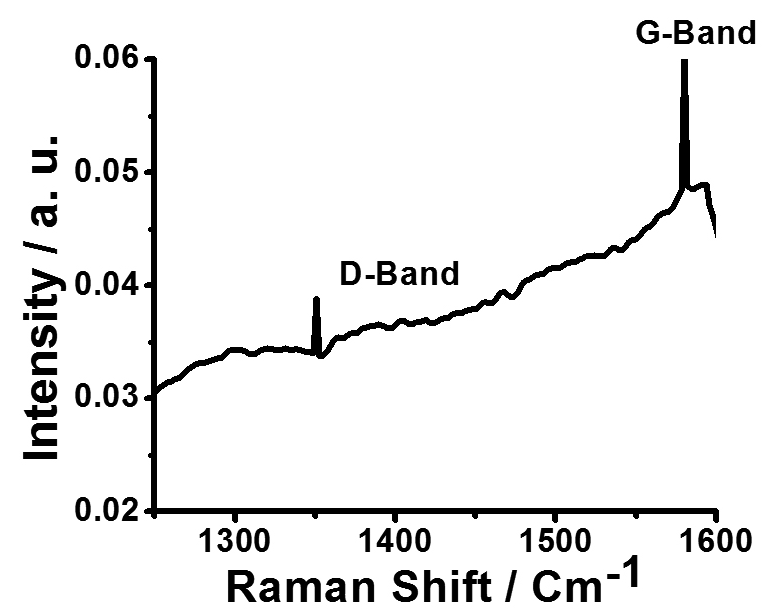

Figure. 3 Raman spectrum of the as- synthesized carbon spheres comprising the peaks at $1380 \mathrm{~cm}^{-1}$ (D-Band) and $1580 \mathrm{~cm}^{-1}$ (G-Band) of graphene structures.

Figure 4 shows the surface morphology of the carbon spheres. The panoramic SEM image in Figure 4(a) reveals that the sample consists of large number of uniform carbon spheres having a smooth surface morphology. Interestingly it can be seen the carbon spheres having 2 to 4 attached lobes and also nodes like structures. These SEM images reveal the formation of about $70-80 \%$ of carbon spheres with polydispersed particle sizes ranging from 200 $\mathrm{nm}$ to $6 \mu \mathrm{m}$.

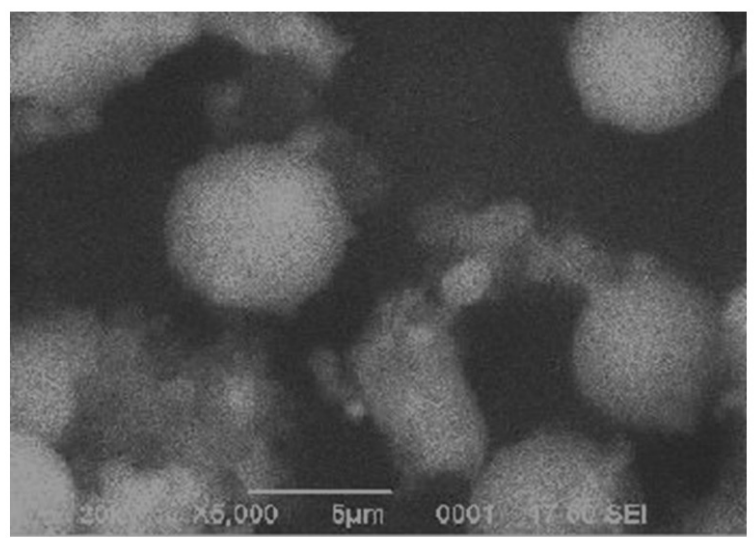

(a)

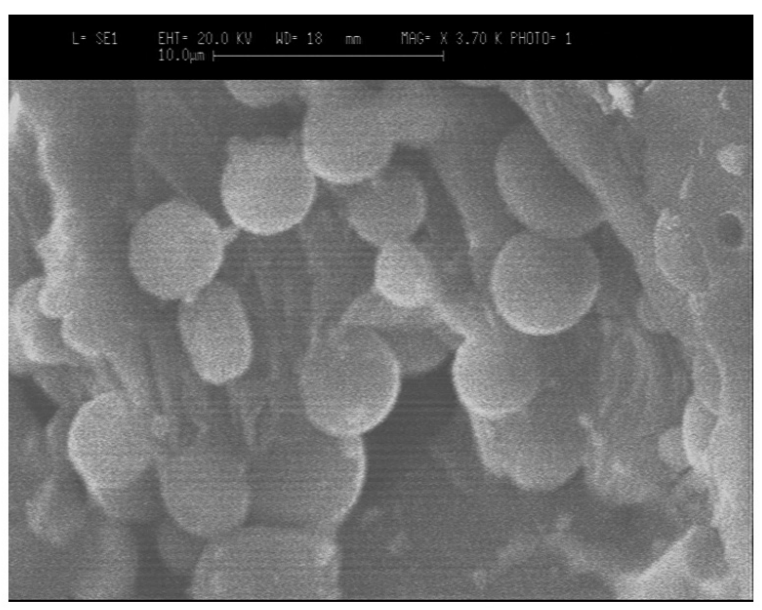

(b) 


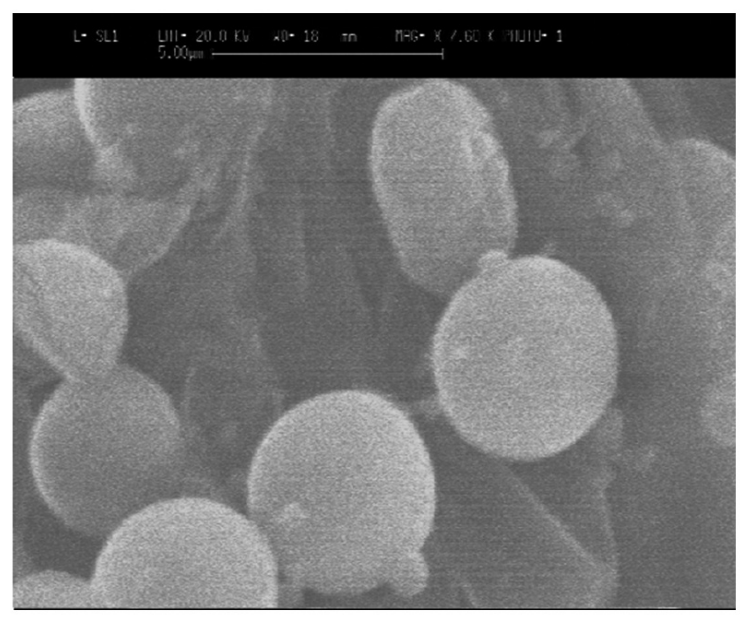

(c)

Figure. 4: (a) (b) and (c) are the SEM images of the as- synthesized carbon spheres at different magnifications.

Figure 5 (a), (b) and (c) represents the TEM images of as obtained carbon spheres. It can be clearly seen from these micrographs that the carbon spheres are having attached lobes on their surface. It can also be observed that carbon spheres are the hard solids and polydispersed in nature. Figure 5 (a) shows carbon sphere having two lobes on top and node like regions on the bottom side. The size of the sphere is in the nano range, which is having a diameter of about $500 \mathrm{~nm}$. Figure 5 (b) depicts the carbon spheres having four prominent lobes almost in tetragonal morphology. This sphere is about $600 \mathrm{~nm}$ in size. The figure 5 (c) shows spheres having porous surface with good porous texture and dia of about $4 \mu \mathrm{m}$. The inset figures in Figure 5 (c) also clearly show the attached lobes on the spheres. A highly porous sponge- or foam-like surface morphology has been observed for these carbon spheres.
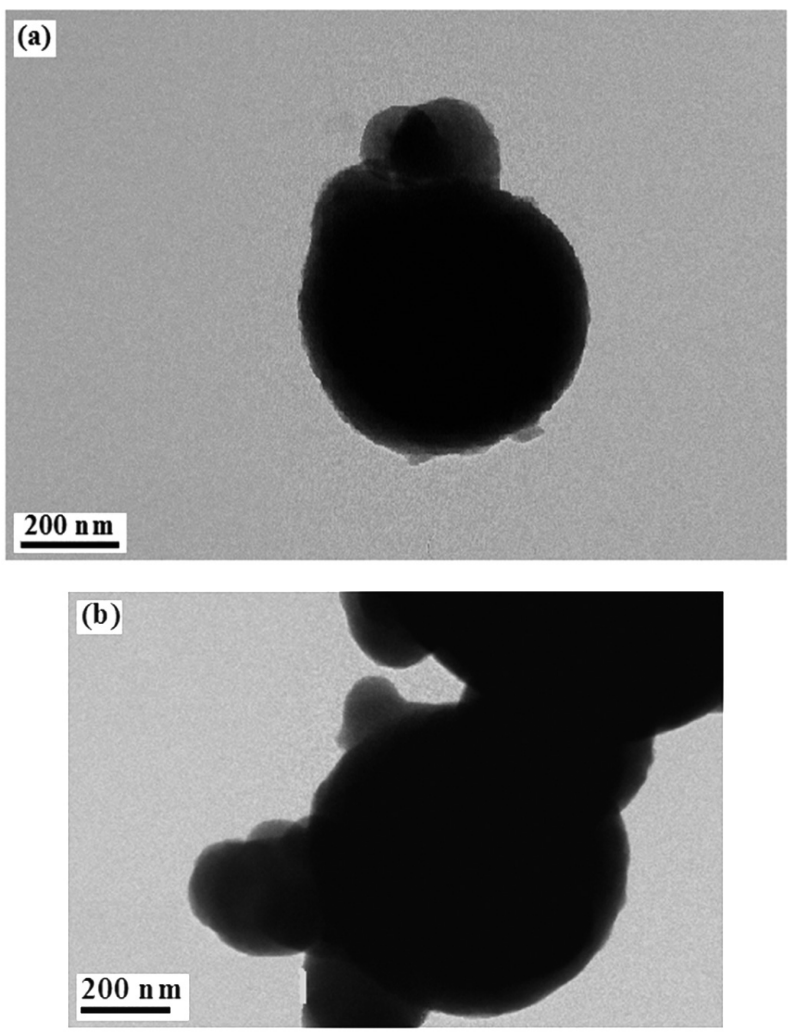

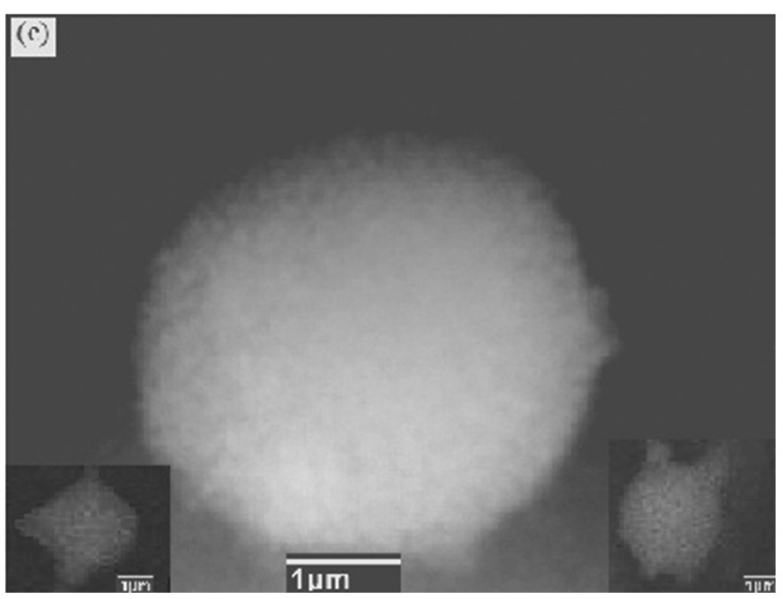

Figure. 5 shows TEM images in which the images (a) and (b) show the nano sized spheres and (c) and (d) show the microsized spheres having lobes and nodes with porous like surface.

The surface area and pore volume of the carbon spheres were obtained by BET-surface analyzer. Nitrogen adsorption-desorption isotherms of these samples were collected at $-196{ }^{\circ} \mathrm{C}$ by using Micomeritics Surface area analyzer model ASAP 2020. Prior to start the analysis, degasing was carried out under vacuum at $2000{ }^{\circ} \mathrm{C}$ for $5 \mathrm{~h}$. The porosity of the carbon particles was characterized using a nitrogen sorption technique. The particles assembled with sucrose display a typical type I isotherm indicating a microporous structure. The specific surface area was calculated using the Brunauer-Emmett-Teller (BET) method based on the adsorption data in the relative pressure $\left(\mathrm{P} / \mathrm{P}_{0}\right)$ range of $0.05-0.25$ from adsorption isotherm branches. The Pore size distribution was calculated by the Barrett-Joyner-Halenda (BJH) method from desorption isotherm branches. The calculated BET surface area and pore volume is 2.956 $\mathrm{m}^{2} \mathrm{~g}^{-1}$ and $0.616 \times 10^{-2} \mathrm{~cm}^{3} \mathrm{~g}^{-1}$ respectively. The observations show that there are two adsorption steps and the first adsorption step at a relative pressure below 0.2 is contributed by the micropores carbon particles and the second adsorption step at a relative pressure above 0.9 is contributed by the mesopores carbon particles. Such a bimodal pore structure is advantageous for applications that require rapid mass transport or pore accessibility to larger molecules.

Earlier, the formation of free elemental carbon in the form of spherical shaped particles occurred by using organic compounds through the decomposition of silicon carbide. ${ }^{29}$ They mentioned that the $\mathrm{C}-\mathrm{O}-\mathrm{H}$ fluids could be the source of formation of diamond and graphite in nature, ${ }^{30,31}$ as well as the theoretical calculations on the $\mathrm{sp}^{2}$ - and $\mathrm{sp}^{3}$-hybridized carbon formation in the $\mathrm{C}-\mathrm{O}-\mathrm{H}$ system encouraged us to explore the use of organic compounds to generate the ideal $\mathrm{C}-\mathrm{O}-\mathrm{H}$ fluid environment. In the present experimental conditions, sucrose first decomposes and then polymerizes to form carbon spheres. ${ }^{32}$ Formation of a sphere probably occurs as it is nucleated from a pentagonal carbon ring followed by a spiral shell growth. When the sphere grows larger, graphitic flakes are nucleated on the surface due to the formation of paired pentagonal-heptagonal carbon rings. ${ }^{12} \mathrm{~A}$ combination of the $\mathrm{P}-\mathrm{H}$ pairs with the hexagonal networks produces eight basic graphitic configurations for forming the sphere, which has been observed experimentally.

The chemistry behind the formation of lobes and nodes over the carbon spheres is not fully understood. However, one of the reasons could be the presence of certain active centers on the surface, which are potential regions for secondary nucleation and growth of the smaller spheres fused to the surface of the main sphere. The nodes like structures are the regions where the formation of lobes was initiated, but ended with incomplete formation. It can be seen that the lobes are mostly formed into axial (binodal), trigonal (trinodal) and tetragonal (tetranodal) shapes. The chemistry behind this is not yet understood. Carbon spheres have properties similar to graphite or fullerene, which allows them to fabricate diamond films, lubricating materials and special rubber additives.

\section{CONCLUSIONS}

Carbon spheres having attractive morphological features such as attached lobes and nodes have been successfully synthesized by simple and cost-effective hydrothermal reactions. This reaction involves the decomposition of sucrose 
in sulfuric acid at a considerably lower temperature of about $200{ }^{\circ} \mathrm{C}$. The product obtained was characterized by X-ray diffraction, FTIR spectroscopy, Raman spectroscopy, SEM and TEM studies. In this work, we have reported a method for the fabrication of novel carbon structures of nano/ micro size from a saccharide at elevated pressure, without the use of any other metal catalyst and template. This method simplifies the preparation of carbon spheres, and makes the process more cost-effective. The morphology, microstructure and surface analyses show that the resultant spheres are solid balls with lobes and nodes having porous surface with diameters ranging from nano to micrometers.

\section{ACKNOWLEDGEMENT}

The Authors would like to acknowledge DST for XRD facility at our Departament. We Would like to acknowledge Prof. P. Vishnu Kamath for his financial support for buying an Oven for our heating experiments. We extend our acknowledgements to EquipTech-AICTE at UVCE, Bangalore 560 001, India, for SEM facility; Prof. Sampath, IPC Departament for Raman spectral runs and Prof. Dhirendra Bhadur, IIT Powai, India, for BET-surface area studies.

\section{REFERENCES}

1. S. Iijima, Nature 354, 56, (1991).

2. B. Ha-da, G. Zhao-xia, Y. Jia, Chin. J. Poly. Sci. 27, 393, (2009).

3. R. S. Ruoff, D. C. Lorents, Carbon 33, 925, (1995).

4. S. Hirano, M. Ozawa, M. Tobinaga, J. Ceram. Soc. 94, 53, (1986).

5. N. Guoqing, L. Yi, W. Fei, W. Qian, L. Guohua, J. Phys. Chem. C. 111, 1969, (2007).

6. H. W. Kroto, J. R. Heath, S. C. Obrien, R. F. Curl, R. E. Smalley, Nature $318,162,(1985)$.

7. A. K. Nebil, U. G. Esteban, A. B. David, G. H. Adria'n, U. Sigita, C. Stefan, Chem. Mater. 19, 2304, (2007).

8. J. W. Liu, M. W. Shao, Q. Tang, X. Y. Chen, Z. P. Liu, Y. T. Qian, Carbon 41, 1682, (2002).

9. B. Y. Liu, D. C. Jia, Q. C. Meng, J. C. Rao, Carbon 45, 668, (2007).

10. L. Q. Xu, W. Q. Zhang, Q. Yang, Y. W. Ding, W. C. Yu, Y. T. Qian, Carbon 43, 1090 (2005).
11. L. Shi, Y. L. Gu, L. Y. Chen, Z. H. Yang, J. H. Ma, Y. T. Qian, Chem. Lett. 33, 532, (2004).

12. Z. L. Wang, Z. C. Kang, J. Phys. Chem. 100, 17725, (1996).

13. Y. B. Ni, M. W. Shao, W. Zhang, Z. C. Wu, Chem. Lett. 3, 494, (2004)

14. W. Qian, L. Z. Wei, F. Y. Cao, Q. W. Chen, Carbon 44, 1303, (2006).

15. Q. H. Zhang, S. Rastogi, D. J. Chen, D. Lippits, P. J. Lemstra, Carbon 44, $778,(2006)$.

16. X. J. Wang, , J. Lu, Y. Xie, G. A. Du, Q. X. Guo, S. Y. Zhang, J. Phys. Chem. B. 106, 933, (2002).

17. J. J. Mack, S. Tari, R. B. Kaner, Inorg. Chem. 45, 4243, (2006).

18. M. Monthioux, Carbon 40, 1809, (2002).

19. X. H. Chen, F. M. Deng, J. X. Wang, H. S. Yang, G. T. Wu, X. B. Zhang, J. C. Peng, W. Z. Li, Chem. Phys. Lett. 336, 201, (2001).

20. C. N. He, N. Q. Zhao, C. S. Shi, X. W. Du, J. J. Li, Mater.Chem. Phys. 97, 109, (2006).

21. S. Yang, X. Chen, S. Motojima, Diamond Relat. Mater. 13, 2152, (2004).

22. B. Basavalingu, K. Byrappa, M. Yoshimura, P. Madhusudan, A. S. Dayananda, J. Mater. Sci. 41, 1465, (2006).

23. J. RobinWhite, Klaus Tauer, Markus Antonietti, Maria-Magdalena Titirici., J. Am. Chem .Soc. 132, 17360, (2010).

24. W. Yong, S. Fabing, L. Jim Yang, X. S. Zhao, Chem. Mater. 18, 1347, (2006).

25. F. H. Yang, R. T. Yang, Carbon, 40, 437, (2002).

26. J. Eric Hampsey, Qingyuan Hu, Lynn Rice, Jiebin Pang, Zhiwang Wu, Yunfeng Lu. Chem. Commun. 3606, (2005).

27. Z. Mingtao, L. Yingliang, X. Yong, Z. Yong, G. Qiu, Y. Dingsheng, J. Phys. Chem. C, 113, 8455, (2009).

28. L. Q. Cheng, Y. L. Liu, J. X. Zhang, D. S. Yuan, C. W. Xu, G. H. Sun, Prog. Chem. 18, 1298, (2006).

29. B. Basavalingu, P. Madhusudan, A. S. Dayananda, K. Lal, K. Byrappa, M. Yoshimura, J. Mater. Sci. 43, 2153, 2008.

30. S. Xu, X. B. Yan, X. L. Wang, S. R. Yang, Q. J. Xuem, J. Mater. Sci, 45, 2619, (2010).

31. P. Lin-Lin, B. Jian-Qiang, B. Yu-Jun, Z. Hui-Ling, Q. Yong-Xin, W. Cheng-Guo, J. Phys. Chem. C. 112, 12134, (2008)

32. X. M. Sun, Y. D. Li, Angew. Chem. 116, 607, (2004). 